Original Article

\title{
Exercise-related fatigue affects joint-driven resistance: comparison of flexor and extensor
}

\author{
Shigeru Terada, RPT, PhD ${ }^{1)^{*}}$, Masahiro Goto, RPT, PhD ${ }^{1)}$, Hiroto Honda, RPT, PhD ${ }^{1)}$, \\ Yoshiniro YAMASHINA, RPT, PhD $^{1)}$
}

1) Department of Physical Therapy, Faculty of Health Science, Aino University: 4-5-4 Higashioda, Ibaraki, Osaka 567-0012, Japan

\begin{abstract}
Purpose] Muscle fatigue can affect the inherent properties of muscles. It is important to know how muscle stiffness changes with muscle fatigue and the different effects of the initial and terminal stages of exercise. Therefore, we aimed to examine the effects of bicep and tricep contraction tasks that lead to fatigue on joint-driven resistance of the elbow joint. [Participants and Methods] Twenty-five healthy men were included. Joint-driven resistance of the elbow joint was measured before and after the muscle contraction task. The slope of the regression line of the angle torque at the time of passive movement was calculated as an elastic coefficient and the entire movable range, proximal range, and distal range were compared. [Results] Owing to the muscular contraction of the biceps and triceps, the elastic coefficient increased in the elbow joint during both flexion and extension. The rate of change in the elastic coefficient was lower during the tricep contraction task than during the bicep contraction task. For both tasks, the change in the elastic coefficient varied depending on the range of exercise. [Conclusion] Resistance exercise increased the driven resistance of the joint during passive movement, and this effect was greater during terminal exercises.

Key words: Muscle fatigue, Elbow joint, Elastic coefficient
\end{abstract}

(This article was submitted Feb. 26, 2021, and was accepted Apr. 7, 2021)

\section{INTRODUCTION}

Muscle fatigue can affect the inherent properties of muscles. Therefore, daily conditioning is important for athletes to prevent muscle fatigue and improve performance. Muscle fatigue is caused by high intensity or prolonged exercise and is known to transiently increase muscle hardness. Muscle fatigue occurs due to microinjury of muscle fibers, increased local blood flow, and increased water transfer between tissues ${ }^{1-3)}$. Massages, stretching exercises, low-intensity exercises, and physical therapy are recommended methods that are used to recover from fatigue ${ }^{4-6)}$. Muscle hardness, circumference, strength, and biochemical index can help determine the effect of these methods. Muscle hardness increases after strength training ${ }^{7}$ ) and it can affect the cross-linked structure (cross-bridges) of actin and myosin at rest ${ }^{8)}$. Muscle stiffness is calculated by measuring angles and joint torque during joint movement. At the beginning of the load, the increase in displacement is small and divided into several phases ${ }^{9}$. It is important to know how muscle stiffness changes before muscle fatigue and the difference between the initial and terminal areas of exercise to ensure faster recovery. In addition, when examining a method for recovering from fatigue, it may be useful to investigate how muscle stiffness is characterized by flexors and extensors. In this study, we investigated how changes in the stiffness of the elbow joint during muscle contraction exercises of the biceps brachii and triceps brachii muscles can lead to fatigue.

*Corresponding author. Shigeru Terada (E-mail: s-terada@pt-u.aino.ac.jp)

(C2021 The Society of Physical Therapy Science. Published by IPEC Inc.

(c) (1) $\odot$ This is an open-access article distributed under the terms of the Creative Commons Attribution Non-Commercial No DerivacC. 


\section{PARTICIPANTS AND METHODS}

The participants were 25 healthy men without neuropathy or osteoarticular disease in the upper limbs. The participants' mean age, height, and weight were $27.9 \pm 8.9$ years, $168.4 \pm 5.8 \mathrm{~cm}$, and $66.1 \pm 9.9 \mathrm{~kg}$ (mean \pm standard deviation), respectively. All participants were right-handed.

This study was performed according to the principles of the Declaration of Helsinki. The purpose and content of the study were explained to all participants in advance, either orally or in writing. It was explained that the obtained data would not be used for any purpose other than research, that personal information would be strictly managed to ensure privacy, and that participation in research was voluntary. All participants understood the content of the study and signed a consent form before participating in the study. This study was conducted after obtaining approval from the Aino University Research Ethics Committee (Aino2019-02).

We used a muscle tonus electromyograph (MTM-06 Muscle Master), manufactured by Medicalnics (Osaka, Japan) to measure elbow joint resistance before and after the muscle contraction task. This device consists of a main unit and a sensor unit; the sensor unit incorporates two upper and lower load cells and a gyro sensor. During passive joint movement, the joint torque and joint angle were measured. First, the participant's weight and forearm length were measured, and the data were entered into the instrument.

The position for the measurement of joint-driven resistance was the chair-sitting position. A highly resilient cushion was placed on the desk, and the participant positioned the elbow in the center of the cushion. The upper limb was positioned in shoulder joint flexion of approximately $60^{\circ}$. The sensor unit was set with the measurement start button positioned at the top, and it was fixed to the participant's proximal wrist joint (Fig. 1).

After 2 minutes of rest, the examiner measured experimental data. Measurements were performed according to instructions on the assist screen of the device. The examiner passively performed flexion and extension movements of the participant's elbow joint five times at a rhythm of once per second. At the time of measurement, the passive movement range was from the maximum extension position of the elbow joint to the maximum flexion angle. Joint angle and torque during passive movement were plotted. The slope $\mathrm{kf}(\mathrm{Nm} / \mathrm{rad})$ of the regression line $(\mathrm{y}=\mathrm{kfx}+\mathrm{b})$, calculated from the plot, reflected the elastic coefficient. In this study, this was used as the measure of joint-driven resistance. Data were analyzed over the entire range of motion, except for the initial and final $10^{\circ}$. Measurement data were calculated by dividing the movable range of the processing target into three types: proximal (bending $60^{\circ}$ to $\left.110^{\circ}\right)$, distal $\left(10^{\circ}\right.$ to $\left.60^{\circ}\right)$, and entire range $\left(10^{\circ}\right.$ to $\left.110^{\circ}\right)$. After measuring the initial joint-driven resistance value, the muscle contraction task was performed.

Muscle contraction tasks were performed with two types of elbow flexion and extension. The elbow flexion task was performed with the right upper limb while sitting in a chair. Using a $5 \mathrm{~kg}$ iron array, the participant repeated the exercise from the maximum extension of the elbow joint to $90^{\circ}$ flexion. The movement speed of flexion/extension was synchronized with the metronome. The movement rhythm was 2 seconds for the return (flexion and extension) movement. The exercise was performed until it became difficult to flex the elbow joint up to $90^{\circ}$ owing to muscle fatigue or until the participant could no longer synchronize the exercise with the rhythm. While the participant continued the exercise task, the examiner confirmed the exercise range and evaluated it at the end of the exercise.

The elbow extension exercise task was performed with the left upper limb in the prone position. The position of the upper limb of the participant was such that the shoulder joint was abducted by $90^{\circ}$. The left forearm was suspended vertically from
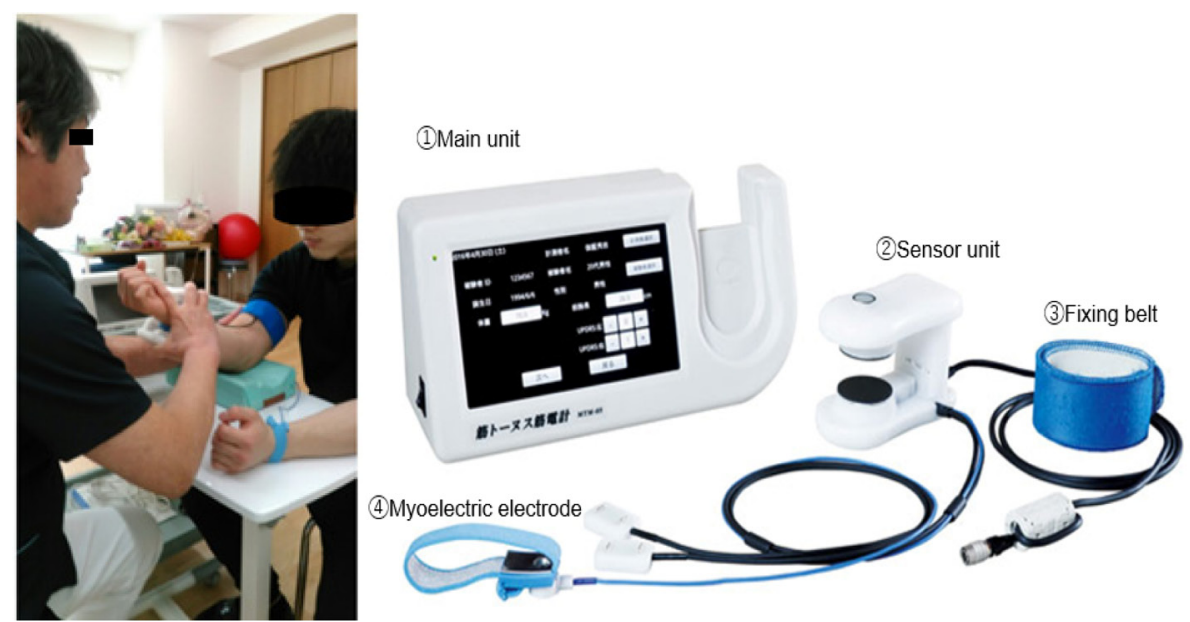

Fig. 1. Measurement position and equipment.

1: Main unit, 2: Sensor unit, 3: Fixing belt, 4: Myoelectric electrode. 
the edge of the bed and placed in an intermediate position between pronation and supination. The participant held the $5 \mathrm{~kg}$ iron array and repeated the movement from the elbow joint in the $90^{\circ}$ flexion position to the maximum extension position. The return (flexion and extension) movement was performed in 2 seconds. The criteria for completing the exercise were the same as those for elbow flexion task.

The two exercise tasks were performed at 30-minute intervals. Immediately after each exercise task, joint-driven resistance was measured again and data before and after the intervention were compared.

Statistical analysis was performed using IBM SPSS Statistics for Windows version 20.0 (IBM Corp., Armonk, NY, USA). First, normality was tested using the Shapiro-Wilk test, then a paired t-test was performed to analyze changes in elastic coefficient before and after the exercise task. The significance level was set at 5\%.

\section{RESULTS}

Figure 2 shows a representative example of the measurement data. The upper part shows an angular torque curve at the time of passive movement of the elbow joint. The $\mathrm{X}$-axis represents the joint angle, and the Y-axis represents the joint torque. The elbow joint passive movement was performed 5 times in 60 seconds, and the angle and torque curve between them are displayed.

Tables 1 and 2 show changes in the elastic coefficient before and after the elbow flexion task. The flexural elastic coefficient was significantly higher in all angle ranges after the exercise than before the exercise. The extension elastic coefficient was similar to the flexion elastic coefficient after the exercise intervention and increased in all angle ranges, with significant differences over the entire range of motion and distal movement.

The rates of increase in the elastic coefficient over the entire range of motion, proximal movement, and distal movement were $15.3 \%, 11.9 \%$, and $22.0 \%$ respectively, for the flexural elastic coefficient, and $14.3 \%, 7.1 \%$, and $28.4 \%$, respectively, for the extension elastic coefficient.

Tables 3 and 4 show the changes in elastic coefficient before and after elbow joint extension tasks. The flexural elastic modulus was higher in all angular ranges after the exercise than before the exercise. The bending elastic coefficient showed a significant difference only in the distal movement. The extension elastic coefficient was the same as the flexion elastic coef-
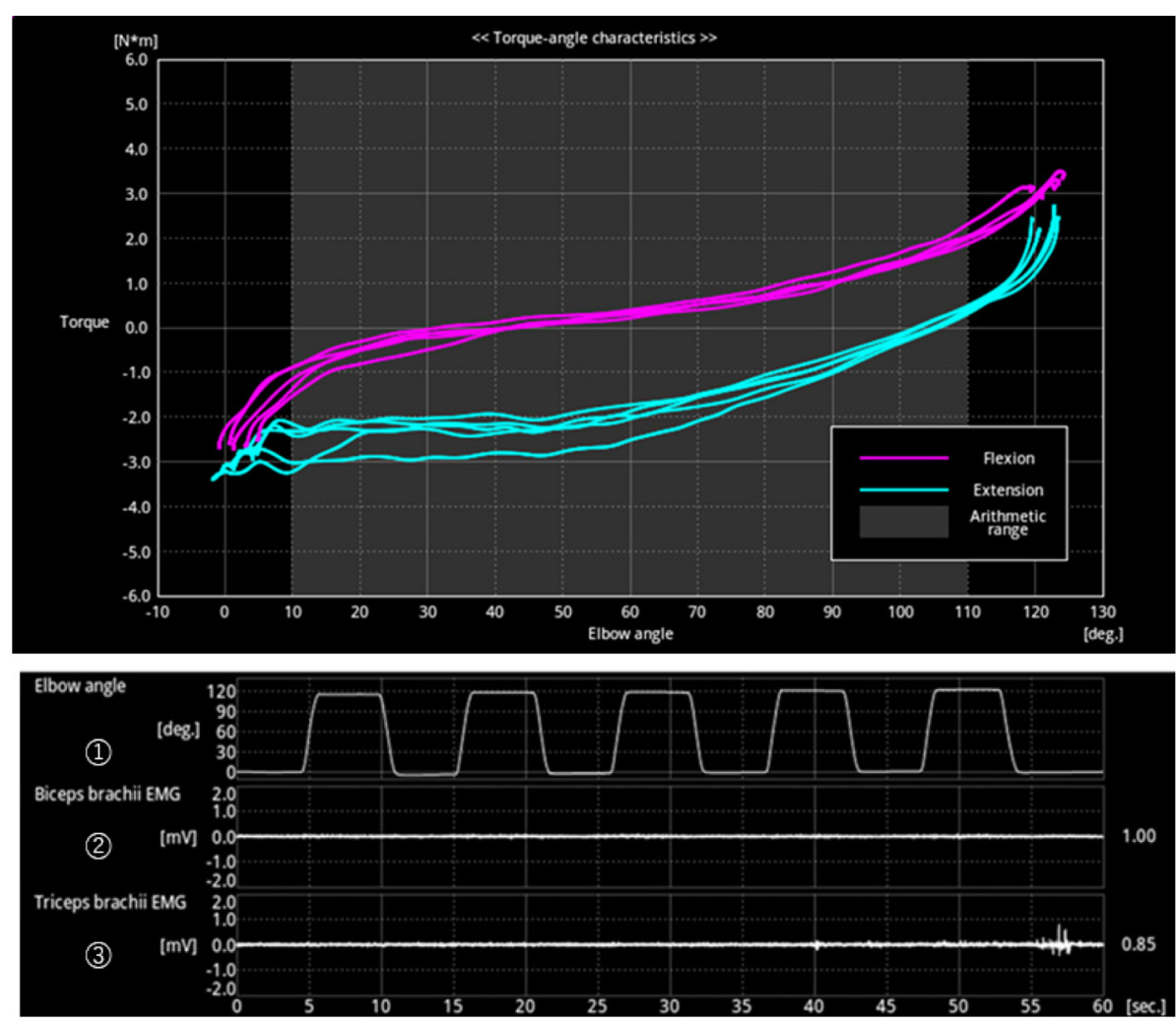

Fig. 2. Representative example of measurement data.

The upper part shows an angular torque curve at the time of passive movement of the elbow joint. The $\mathrm{X}$-axis represents the joint angle, and the Y-axis represents the joint torque. The lower part shows 1: the angle change of the elbow joint and the electromyogram of 2: biceps brachii and 3: during measurement. 
Table 1. Comparison of flexion elastic coefficients before and after elbow flexion tasks ( Nm/rad)

\begin{tabular}{lccc}
\hline & Before intervention & After intervention & $\mathrm{p}$-value \\
\hline Total range of motion & $0.85 \pm 0.24$ & $0.98 \pm 0.25$ & $\mathrm{p}<0.001^{* *}$ \\
Proximal & $1.26 \pm 0.37$ & $1.41 \pm 0.64$ & $0.040^{*}$ \\
Distal & $0.59 \pm 0.31$ & $0.72 \pm 0.39$ & $0.011^{*}$ \\
\hline
\end{tabular}

Values are mean \pm standard deviation. ${ }^{*} \mathrm{p}<0.05,{ }^{* *} \mathrm{p}<0.01$.

Table 2. Comparison of extension elastic coefficients before and after elbow flexion tasks ( $\mathrm{Nm} / \mathrm{rad})$

\begin{tabular}{lccc}
\hline & Before intervention & After intervention & $\mathrm{p}$-value \\
\hline Total range of motion & $0.84 \pm 0.26$ & $0.96 \pm 0.26$ & $\mathrm{p}<0.001^{* *}$ \\
Proximal & $1.12 \pm 0.40$ & $1.20 \pm 0.45$ & 0.113 \\
Distal & $0.67 \pm 0.27$ & $0.86 \pm 0.34$ & $0.008^{* *}$ \\
\hline
\end{tabular}

Values are mean \pm standard deviation. ${ }^{* *} \mathrm{p}<0.01$.

Table 3. Comparison of flexion elastic coefficients before and after the elbow joint extension task $(\mathrm{Nm} / \mathrm{rad})$

\begin{tabular}{lccc}
\hline & Before intervention & After intervention & p-value \\
\hline Total range of motion & $0.95 \pm 0.25$ & $1.00 \pm 0.25$ & 0.052 \\
Proximal & $1.51 \pm 0.51$ & $1.52 \pm 0.50$ & 0.824 \\
Distal & $0.50 \pm 0.23$ & $0.58 \pm 0.24$ & $0.039^{*}$ \\
\hline
\end{tabular}

Values are mean \pm standard deviation. ${ }^{*} \mathrm{p}<0.05$.

Table 4. Comparison of extension elastic coefficients before and after the elbow extension task ( $\mathrm{Nm} / \mathrm{rad})$

\begin{tabular}{lccc}
\hline & Before intervention & After intervention & p-value \\
\hline Total range of motion & $0.99 \pm 0.28$ & $1.01 \pm 0.33$ & 0.524 \\
Proximal & $1.36 \pm 0.50$ & $1.38 \pm 0.53$ & 0.694 \\
Distal & $0.72 \pm 0.32$ & $0.79 \pm 0.36$ & 0.362 \\
\hline
\end{tabular}

Values are mean \pm standard deviation.

ficient after exercise intervention, as it increased in all angles. However, there was no significant difference in the extension elastic coefficient in all angle ranges.

The rates of increase in the entire range of motion, proximal movement, and distal movement were 5.3\%, $0.6 \%$, and $16.0 \%$, respectively, in the flexion elastic coefficients, and $2.0 \%, 1.5 \%$, and $9.7 \%$, respectively, in the extension elastic coefficients.

\section{DISCUSSION}

Contraction leading to fatigue of the biceps and triceps, increased the elastic coefficient in both flexion and extension movements of the elbow joint. Previous reports found that after exercise it took approximately 8 minutes for the intramuscular pressure to become constant owing to temporary muscle hardening caused by muscle activity ${ }^{10)}$. This hardening is caused by an increase in local blood flow in muscles and water transfer between tissues ${ }^{1,3)}$. Increased blood flow during muscle contraction increases the capillary bed owing to dilation of arterioles and the anterior capillary sphincter. In addition, an increase in the hydrostatic pressure in the capillaries increases the amount of water in the tissue gap. The accumulation of excess tissue water in the myocytes' interstitial space increases the intramuscular pressure, resulting in post-exercise muscle hardening and high volume ${ }^{11,12)}$. Therefore, water retention in the cell gap causes an increase in the intramuscular pressure, which causes muscle swelling and, consequently, an increase in joint resistance. Hydrogen ion concentration is elevated during muscle fatigue and reduces the uptake of calcium ions into the sarcoplasmic reticulum; this prolongs the muscle relaxation time ${ }^{13)}$. It is also possible that passive resistance of the joints increases owing to the extension resistance of the muscles applied during exercise, caused by suppression of muscle relaxation.

The elastic coefficient of flexion and extension of the elbow joint increased owing to the contraction of the biceps muscle, and significant differences were observed in the extension elastic coefficient, except for the proximal movement. In addition, the rate of increase in the elastic coefficient was higher in the distal region than in the proximal region. First, we considered 
joint-driven resistance in the extension movement. The rate of increase in the extension elastic coefficient was the greatest at the distal end. In this range, the elbow joint was bent by $10^{\circ}-60^{\circ}$. A previous report showed that hardness of the biceps brachii muscle peaks at full extension and then gradually decreases to a minimum at $60^{\circ 14)}$. With the resting length as the boundary, the muscle shifts from stretching to shortening, and the resting tension decreases. In addition, Noda et al. ${ }^{15)}$, reported that the viscosity constant of the muscle increased significantly before and after isometric muscle contraction. Accordingly, we considered that, because of the increase in muscle viscosity and the hardness of the biceps brachii muscle, which is a dynamic muscle, passive resistance of the joint increased the most in the distal range where the muscle was stretched.

The increase in the driven resistance in the elbow joint bending direction should be considered. The rates of change in both the flexion and extension elastic coefficient were the greatest at the distal end. However, the degree of change in the flexion elastic coefficient was smaller than that in the extension elastic coefficient. Additionally, the elastic coefficient increased after muscle contraction even at the proximal side. The bending direction is the direction in which the biceps of the upper arm bends while shortening. Initially, we expected that there would be no increase in exercise resistance during flexion. However, our results showed the opposite, indicating that the increase in viscosity and stiffness of the biceps muscle also affected the resistance to movement in the direction of muscle shortening. This suggested that contraction of dynamic muscles affected exercise resistance in both directions, i.e., muscle extension and flexion. This may be due to the increased resistance to sarcomere slipping in the biceps. The completion criteria for the muscle contraction task were when the elbow joint was unable to flex and extend from $0^{\circ}$ to $90^{\circ}$. However, in the latter half of the exercise, excessive effort may have resulted in compensatory movements and muscle contraction of the triceps, which should be the antagonist. Generally, joint movement causes a relative increase in antagonistic muscle activity with an increase in the angular velocity. In successive repetitive movements, muscle output is offset or added to by antagonists ${ }^{16}$. Electromyography was not performed during the muscle contraction task in this study, and it may be necessary to improve the intervention method by altering the exercise speed.

The elastic coefficient of flexion and extension of the elbow joint appeared to increase owing to contraction of the triceps muscle, and significant differences were observed only in the distal part of the flexion elastic coefficient. The rate of change in the elastic coefficient for triceps contraction was generally smaller than that in the elastic coefficient for biceps contraction. This result indicated that passive resistance of the joint increased in the initial range of the extension of the triceps brachii. This differed from the assessment findings of the biceps muscle contraction task.

Each muscle has its characteristic shape and muscle fiber composition. The biceps brachii is spindle-shaped, while the triceps brachii is a pennate muscle. The physiological cross-sectional area of the triceps brachii is larger than that of the biceps brachii, but the muscle fiber length is shorter ${ }^{17}$. During passive movement, muscles that antagonize joint movement are stretched. When the direction of the long axis of the muscle and the direction of the muscle fiber match, the external force acting on the muscle extension is applied straight to the muscle. This is because the biceps brachii is a parallel muscle. In addition, the muscle fiber length of the biceps brachii is 2.5 times longer than that of the triceps brachii ${ }^{18)}$, and its range of expansion and contraction is large. Since the triceps brachii is a pennate muscle, the direction of the long axis of the muscle and the direction of the muscle fiber do not match. Therefore, the stretching force acting on the muscle may be reduced by the pennate angle. This difference in muscle shape and muscle fiber length may have affected the resistance to stretching the muscle. However, we did not examine muscle stretch dynamics in this study. Therefore, in the future, it will be necessary to measure the stretched state of muscles using ultrasonic waves.

Another factor that may have influenced our results is the amount of exercise load. The exercise intervention task started with the biceps, followed by the triceps exercise task. A 30 minute break was taken between measurements. Nevertheless, the two tasks were performed on the same day, which may have affected participant motivation. Consequently, the muscular contraction task of the triceps may have had a smaller load than that of the biceps. Lieber ${ }^{19}$, also reported that in the forcevelocity relationship, muscles with long muscle fibers shift toward velocity and muscles with a large muscle cross-sectional area shift toward force. The contraction task of the triceps brachii was performed using elbow extension in the prone position. The exercise speed at this time had the same rhythm as that of the exercise with the biceps brachii. It may have been necessary to consider the difference in muscle composition between the biceps brachii and triceps brachii when determining the exercise speed and exercise method.

This study had some limitations. Muscle mass, muscle cross-sectional area, and muscle length may affect muscle stiffness. Therefore, the participants' body composition should be considered when analyzing data. In addition, passive flexion and extension at the elbow joint were performed five times to measure muscle stiffness, and the average value was used. Furthermore, the elastic coefficient after the muscle contraction task may be affected by muscle contraction in the first and second movements. Therefore, in the future, the first to fifth elastic coefficients should be analyzed individually, including the persistence of the effect on stiffness.

\section{Conflict of interest}

The authors have no conflicts of interest directly relevant to the content of this article. 


\section{REFERENCES}

1) Lundvall J, Mellander S, Westling H, et al.: Fluid transfer between blood and tissues during exercise. Acta Physiol Scand, 1972, 85: 258-269. [Medline] [CrossRef]

2) Kagaya A, Homma S: Brachial arterial blood flow during static handgrip exercise of short duration at varying intensities studied by a Doppler ultrasound method. Acta Physiol Scand, 1997, 160: 257-265. [Medline] [CrossRef]

3) Murayama M, Nosaka K, Yoneda T, et al.: Changes in hardness of the human elbow flexor muscles after eccentric exercise. Eur J Appl Physiol, 2000, 82: 361-367. [Medline] [CrossRef]

4) Cafarelli E, Flint F: The role of massage in preparation for and recovery from exercise. An overview. Sports Med, 1992, 14: 1-9. [Medline] [CrossRef]

5) McNair PJ, Stanley SN: Effect of passive stretching and jogging on the series elastic muscle stiffness and range of motion of the ankle joint. Br J Sports Med, 1996, 30: 313-317, discussion 318. [Medline] [CrossRef]

6) Gorgey AS, Wadee AN, Sobhi NN: The effect of low-level laser therapy on electrically induced muscle fatigue: a pilot study. Photomed Laser Surg, 2008, 26: 501-506. [Medline] [CrossRef]

7) Matsubara Y, Awai H, Kimura G, et al.: Effect of vibration stimulation on recovery of muscle stiffness after isometric muscle contraction had achieved fatigue. Rigakuryoho Kagaku, 2004, 19: 341-345. [CrossRef]

8) Seki M: Biomechanical properties during passive ankle movement in spastic hemiplegic patients. Jpn J Rehabil Med, 2001, 38: 259-267. [CrossRef]

9) Kuitunen S, Komi PV, Kyröläinen H: Knee and ankle joint stiffness in sprint running. Med Sci Sports Exerc, 2002, 34: 166-173. [Medline] [CrossRef]

10) Arimizu J, Ogata K, Naito M, et al.: Measurement of intracompartmental pressure, before, during and after exercise. Orthop Traumatol, 1993, 42: 1056-1058. [CrossRef]

11) Sadamoto T, Bonde-Petersen F, Suzuki Y: Skeletal muscle tension, flow, pressure, and EMG during sustained isometric contractions in humans. Eur J Appl Physiol Occup Physiol, 1983, 51: 395-408. [Medline] [CrossRef]

12) Sejersted OM, Hargens AR, Kardel KR, et al.: Intramuscular fluid pressure during isometric contraction of human skeletal muscle. J Appl Physiol, 1984, 56: 287-295. [Medline] [CrossRef]

13) Schwellnus MP, Derman EW, Noakes TD: Aetiology of skeletal muscle 'cramps' during exercise: a novel hypothesis. J Sports Sci, 1997, 15: 277-285. [Medline] [CrossRef]

14) Nakano M, Tsunoda N: Is muscle stiffness measurement affected on different athletic training in human elbow flexors. Kokushikan Soc Sport Sci, 1997, 2: 19-23 [in Japanese].

15) Noda M, Shibayama A: Change of viscoelastic properties in human triceps surae after isometric endurance exercise. JJBSE, 2000, 4: 232-243.

16) Takayanagi K, Ihara H: A comparison with eccentric and concentric muscular contraction on flexors and extensors in the normal human knee using muscular strength and EMG. Phys Ther Jpn, 1992, 19: 30-35.

17) Edgerton VR, Roy RR: Specific tension of human elbow flexor muscle. In: Biochemistry of exercise VI. Champaign: Human Kinetics, 1986, pp 487-500.

18) Kawakami Y, Nakazawa K, Fujimoto T, et al.: Specific tension of elbow flexor and extensor muscles based on magnetic resonance imaging. Eur J Appl Physiol Occup Physiol, 1994, 68: 139-147. [Medline] [CrossRef]

19) Lieber RL, Fridén J: Clinical significance of skeletal muscle architecture. Clin Orthop Relat Res, 2001, 383: 140-151. [Medline] [CrossRef] 